\title{
Die Zukunft der Public Health-Forschung in Deutschland
}

Autoren: A. Gerhardus, B. Babitsch, B. Blättner, G. Bolte, I. Brandes, T. Gerlinger (Vorstand der Deutschen Gesellschaft für Public Health)

\section{Bibliografie}

DOI http://dx.doi.org/ $10.1055 / s-0032-1306318$ Gesundheitswesen 2012; 74: $121-122$

(c) Georg Thieme Verlag KG Stuttgart $\cdot$ New York

ISSN 0941-3790

\section{Korrespondenzadresse}

Prof. Dr. med. A. Gerhardus, M.A., MPH

Abteilung 1: Versorgungsforschung, Institut für Public Health und Pflegeforschung, FB 11

Universität Bremen

Grazer Straße 4

28359 Bremen

ansgar.gerhardus@uni-bremen.de
Spricht man Menschen in Peru auf Malariaprävention an, kann das zu Missverständnissen führen: „La Malaria“ sind für sie Trupps uniformierter Männer, die bis Anfang der 1990er Jahre in die Dörfer fuhren, um DDT zu versprühen, denn auf ihren Autos prangte in großen Lettern das Wort „Malaria“. Bereitwillig räumten die Menschen für das Sprühen durch „la Malaria“ ihre Häuser leer - das reduzierte die Zahl der lästigen Mückenstiche drastisch. Eine Verbindung mit der Krankheit „paludismo“ (lokaler Begriff für Malaria) stellte dagegen kaum jemand her.

Ähnliches passierte in Deutschland noch vor 25 Jahren mit dem Begriff „Öffentliche Gesundheit“. Die meisten Menschen verbanden damit grauhaarige Männer in weißen Kitteln, die Größe und Gewicht von schulpflichtigen Kindern erfassten. Vergleichbar zu Peru, wo Malaria mit einer hierarchisch strukturierten, vom sonstigen Umfeld losgelösten staatlichen Einheit verbunden wurde, galt in Deutschland „die Öffentliche Gesundheit“ als ein (langweiliger) hochspezialisierter, staatlich-medizinischer Dienst, in dem Normalbürger keine aktive Rolle spielten.

Als in Deutschland der Fokus von Öffentlicher Gesundheit noch auf zentralbürokratisch geplanten, technischen Programmen und Erziehungskampagnen lag, hatte Public Health in anderen Ländern eine viel breitere, oft auch gesellschaftsund systemkritische Bedeutung. In Großbritannien startete bereits 1967 die erste der Whitehall-Studien, in denen der Zusammenhang zwischen Sozialstatus und Mortalität untersucht wurde (http://www.ucl.ac.uk/whitehallI//history). Heute sind sozial bedingte Ungleichheiten in der Gesundheit weithin anerkannt und Wege, diese zu reduzieren, gut beschrieben, wie bspw. in den Berichten der WHO-Kommission zu sozialen Determinanten für Gesundheit (http://www.who. int/social_determinants/en/). In den USA entdeckte Jack Wennberg mit seinem Team in den frühen 70er Jahren eine erstaunliche Variation in der Gesundheitsversorgung zwischen verschiedenen geografischen Regionen, die sich nicht mit einem unterschiedlichem Bedarf, wohl aber durch Unterschiede in der technischen Infrastruktur erklären ließ [1]. Ebenfalls in den USA untersuchten Susan Sontag, Arthur Kleinman und andere die Bedeutung von Kultur und Geschlecht für das Erleben und die Repräsentation von Krankheit und Gesundheit und wurden zu Wegbereitern eines emanzipatorischen Verständnisses von Public Health [2,3]. Andere Länder hatten Deutschland überholt - trotz eines ursprünglichen Vorsprungs dank herausragender
Persönlichkeiten wie Johann Peter Frank und Rudolf Virchow. Deren Arbeiten zu sozialen, politischen und ökonomischen Einflussfaktoren auf die Gesundheit hatten die Entwicklungen in Europa im 18. und 19. Jahrhundert maßgeblich geprägt.

Dies änderte sich Ende der 1980er Jahre mit der Förderung der Forschungsverbünde Public Health durch das Bundesministerium für Bildung und Forschung (BMBF). Die damalige Institutionalisierung von Public Health war eine Erfolgsgeschichte: In nur fünf Jahren entstanden neun Masterstudiengänge und Public Health-Institute, weitere sind dazugekommen. Nach bemerkenswert kurzer Zeit kam es zu Spezialisierungen und „Ausgründungen“: Disziplinen wie Epidemiologie, Versorgungsforschung oder Gesundheitsökonomie entwickelten rasch ihre eigenen Profile und wurden weitgehend unabhängig. Spezialisierte Lehrstühle und Fachgesellschaften entstanden, verbunden mit einem breit gefächerten Feld an Studienmöglichkeiten. Diese Entwicklung trug schnell erste Früchte: Angeregt durch die Arbeiten von Jack Wennberg zeigte der Bericht des Sachverständigenrats (SVR) zu „Über-, Unter-, Fehlversorgung“ eindrückliche Mängel auf und prägte die Diskussion zur Gestaltung des Gesundheitswesens bis heute [4]. Studien zur Gesundheit am Arbeitsplatz, zum Einfluss von Flug- oder Straßenlärm auf die Gesundheit, zu den Effekten von klimatischen Veränderungen und zu den sozialen Determinanten von Gesundheit hatten und haben Einfluss weit über den eigentlichen Gesundheitssektor hinaus. Public Health in Deutschland hat innerhalb einer Dekade in vielen Bereichen (wieder) Anschluss an die internationale Forschung gefunden und wirkt von dort auf Politik und Praxis ein. Durch die Ausdifferenzierung werden einige der Erfolge aber nicht Public Health, sondern den Einzeldisziplinen zugeschrieben.

Der Bedarf an wissenschaftlich geprägten Public Health-Strategien hat deutlich zugenommen: Innerhalb der letzten 100 Jahre kam es zu einer nachhaltigen Verschiebung in der Krankheitslast. Dominierten im 19. Jahrhundert Infektionskrankheiten Morbidität und Mortalität, stehen heute chronische Erkrankungen im Vordergrund. Trotz des langen Zeitraums sind die Gesundheitssektoren, in Deutschland wie auch international, auch heute noch weit davon entfernt, angemessen auf diese (nicht-so-) neuen, spezifischen Herausforderungen zu reagieren.

Strategien, die zur Kontrolle von Infektionskrankheiten einen Teil des Erfolges ausmachten, 
wie zentrale „Gesundheitserziehungsprogramme“, können für die Prävention komplexer chronischer Erkrankungen völlig ungeeignet sein. Ein Beispiel: Aus Gesundheitssurveys, z.B. des Robert Koch-Instituts, ist bekannt, dass Übergewicht in unterschiedlichen ethnischen und sozialen Gruppen unterschiedlich verteilt ist. Wir wissen auch, dass die Ursachen, die zu Übergewicht führen zwischen den Gruppen stark variieren können. Ebenfalls ist gut belegt, dass öffentliche Appelle wie „weniger essen, mehr bewegen“ die am schwersten Betroffenen in der Regel nicht erreichen, diese aber dadurch stärker stigmatisiert werden. An diesen Punkten setzt Public Health-Forschung an und entwickelt differenzierte Modelle und Strategien, um auf diese Herausforderungen zu reagieren. Im Rahmen dieses Prozesses wurde nicht zuletzt die überragende Bedeutung der sozialen Lage für die Gesundheit wiederentdeckt; zunehmend in den Fokus rücken Einflüsse aus der natürlichen und sozialen Umwelt einschließlich der Arbeitswelt.

Vor diesem Hintergrund haben die Mitglieder der Deutschen Gesellschaft für Public Health im September 2011 den Vorstand beauftragt, ein Memorandum zur Zukunft der Public HealthForschung in Deutschland vorzulegen. Die Fertigstellung erfolgt im September 2012, wichtige strukturelle Probleme der staatlichen Förderung von Public Health-Forschung wurden aber bereits jetzt deutlich:

Public Health ist ein Ziel, die Verbesserung der Gesundheit und die Prävention von Krankheiten, zu dem viele verschiedene Disziplinen beitragen. Förderstrukturen sind dagegen disziplinär ausgerichtet, woran innovative und notwendige Forschungsvorhaben oft scheitern. Beispielsweise existieren für ein wichtiges Public Health-Thema wie Übergewicht soziale, biologische, ökonomische, politische und kulturelle Kausalmodelle. Jedes Modell ist mit eigenen Theorien, Interventionen und Erfolgsparametern verknüpft. Public Health bildet einen Rahmen, in dem sich die unterschiedlichen Modelle ergänzen und in unterschiedlichen Kontexten und Situationen jeweils auf verschiedene Weise nutzbar gemacht werden können. In den gegenwärtigen Förderstrukturen wird dieser Ansatz nicht ausreichend abgebildet. Soweit Anträge die mehrere Modelle umfassen überhaupt in disziplinär gedachte Ausschreibungen hineinpassen, laufen sie Gefahr zwischen sozialwissenschaftlich, naturwissenschaftlich und medizinisch geprägten Gutachter/innen hin- und hergeschoben zu werden, mit unterschiedlichen und zum Teil widersprüchlichen Kriterien und Rückmeldungen. In einem angemessenen Reviewverfahren müsste die Gruppe der Gutachter/innen die fachliche und epistemologische Breite dieser Anträge widerspiegeln und sich untereinander verständigen, um widersprüchliche Kriterien und Empfehlungen zu vermeiden und in Systemzusammenhängen zu denken.

Förderlinien werden oft auch nach spezifischen Erkrankungen definiert. Tatsächlich können eng gefasste top-down organisierte Interventionen gegen definierte Indikatoren spezifischer Erkrankungen sehr effektiv sein. Public Health-Interventionen gehen aber oft über die Veränderung einzelner Indikatoren hinaus. Eine Intervention, die auf die Verbesserung gesundheitlicher Teilhabe benachteiligter Bevölkerungsgruppen zielt, wird im Vergleich zu einem oralen Antidiabetikum schlechter abschneiden, wenn lediglich spezifische Indikatoren z. B. der HbA1c-Wert berücksichtigt werden. Dabei bleibt außer Acht, dass eine Intervention zur Verbesserung der Teilhabe Einfluss auf andere Er- krankungen und Indikatoren, soziale wie auch medizinische, haben kann. Dieser mögliche Gewinn befindet sich jedoch außerhalb des Blickfelds krankheitsspezifischer Ausschreibungen. Gerade aktuelle Förderprogramme zielen bevorzugt auf neuere technische Entwicklungen ab, wie z. B. genetisches Testen, bildgebende Verfahren oder E-Health. Begründet wird das häufig mit einer Kombination aus möglichen gesundheitlichen Gewinnen und wirtschaftlichem Potential. Ein expliziter Nachweis wird dann weder für den einen noch für den anderen Bereich erwartet. Soziale Innovationen, wie z. B. veränderte Arbeits- und Bildungsstrukturen oder eine quartiersbezogene Gesundheitsförderung, spielen dagegen eine vernachlässigbare Rolle. Public Health mit ihren Spezialgebieten wird in diesen Förderprogrammen zur Begleitforschung marginalisiert, bevorzugt mit dem Ziel zur Verbesserung der Akzeptanz und der Dissemination der Technologien beizutragen.

Wie sind Stand und die Perspektive der Public Health-Forschung im Jahr 2012? Genau gegenläufig zum steigenden Bedarf hat sich die staatliche Förderpolitik entwickelt. In den entscheidenden Rahmenpapieren der letzten Jahre zur gesundheitlichen Forschungsförderung des BMBF, der „Roadmap“ und dem „Rahmenprogramm Gesundheitsforschung“ kommt „Public Health“ nicht einmal als Begriff vor $[5,6]$. Anders als in den biowissenschaftlich geprägten Disziplinen kann dieser Mangel aufgrund der fehlenden gewinnorientierten Verwertbarkeit nicht durch industrielle Förderung kompensiert werden.

Dem gegenüber ist in den USA die Lage der Public Health-Forschung wesentlich günstiger. Die Zahl der „Schools of Public Health“, ist, bezogen auf die Einwohnerzahl, weiterhin deutlich höher als in Deutschland. Für die gesundheitliche Forschungsförderung, die nicht nach Disziplinen, Krankheiten, oder technischen Innovationen, sondern nach gesundheitsbedarfsbezogenen Kriterien priorisiert wurden, sind seit 2009 mit großem Erfolg Programme in Milliardenhöhe aufgelegt worden [7]. Ohne eine rasche Kurskorrektur besteht die Gefahr, dass die Public Health-Forschung in Deutschland ein zweites Mal den internationalen Anschluss verpasst.

\section{Literatur}

1 Wennberg J, Gittelsohn A. Small area variations in health care delivery. Science 1973; 182 (4117): 1102-1108

2 Kleinman A. Patients and healers in the context of culture: an exploration of the borderland between anthropology, medicine, and psychiatry. University of California Press; Berkeley and Los Angeles: 1980

3 Sontag S. Illness as metaphor. Farrar, Straus and Giroux; New York: 1978

4 Sachverständigenrat für die Konzertierte Aktion im Gesundheitswesen (SVR). Gutachten 2000/2001 des SVR. Bedarfsgerechtigkeit und Wirtschaftlichkeit. Band III. Über-, Unter- und Fehlversorgung. Drucksache 14/6871. http://dip21.bundestag.de/dip21/btd/14/068/1406871.pdf Zugriff am 14.2.2012

5 Gesundheitsforschungsrat (GFR) des BMBF, Hrsg. Roadmap für das Gesundheitsforschungsprogramm der Bundesregierung. Bonn, Berlin: GFR; 2007 http://www.bmbf.de/pubRD/roadmap_Gesundheitsfor schung07_lang.pdf Zugriff am 14.2.2012

$6 B M B F$, Hrsg. Rahmenprogramm Gesundheitsforschung der Bundesregierung. Bonn, Berlin: BMBF; 2010 http://www.bmbf.de/pub/gesund heitsforschung.pdf Zugriff am 14.2.2012

7 Federal Coordinating Council for Comparative Effectiveness Research. Report to the President and the Congress. Washington: U.S. Department of Health \& Human Services; 2009 http://www.hhs.gov/recove ry/programs/cer/cerannualrpt.pdf Zugriff am 14.2.2012 